\title{
Rips construction and Kazhdan property $(T)$
}

\author{
Igor Belegradek and Denis Osin*
}

\begin{abstract}
We show that for any non-elementary hyperbolic group $H$ and any finitely presented group $Q$, there exists a short exact sequence $1 \rightarrow N \rightarrow G \rightarrow Q \rightarrow 1$, where $G$ is a hyperbolic group and $N$ is a quotient group of $H$. As an application we construct a hyperbolic group that has the same $n$-dimensional complex representations as a given finitely generated group, show that adding relations of the form $x^{n}=1$ to a presentation of a hyperbolic group may drastically change the group even in case $n \gg 1$, and prove that some properties (e.g. properties (T) and FA) are not recursively recognizable in the class of hyperbolic groups. A relatively hyperbolic version of this theorem is also used to generalize results of Ollivier-Wise on outer automorphism groups of Kazhdan groups.
\end{abstract}

Mathematics Subject Classification (2000). 20F67, 20F65, 20 F28.

Keywords. Hyperbolic group, Rips construction, Kazhdan property (T).

\section{Introduction and main results}

Given any finitely presented group $Q$, the Rips construction [33] produces a short exact sequence

$$
1 \rightarrow N \rightarrow G \rightarrow Q \rightarrow 1,
$$

where $G$ is a hyperbolic group and $N$ is finitely generated. This result and its variations are a powerful source of pathological examples in geometric group theory, see [4], [9], [37], [24]. In this note the small cancellation methods developed in [28] and [1] are used to prove the following version of the Rips construction. (We refer to the next section for definitions.)

Theorem 1.1. Let $H$ be a non-elementary hyperbolic group, $Q$ a finitely generated group, $S$ a subgroup of $Q$. Suppose that $Q$ is finitely presented with respect to $S$. Then there exists a short exact sequence

$$
1 \rightarrow N \rightarrow G \stackrel{\varepsilon}{\rightarrow} Q \rightarrow 1,
$$

\footnotetext{
*The authors were supported by the NSF grants DMS-0503864 (Belegradek) and DMS-0605093 (Osin).
} 
and an embedding $\iota: S \rightarrow G$ such that

(a) $N$ is isomorphic to a quotient group of $H$.

(b) $G$ is hyperbolic relative to the (proper) subgroup $\iota(S)$.

(c) $\iota \circ \varepsilon \equiv$ id.

(d) If $H$ and $Q$ are torsion free, then so is $G$.

(e) The canonical map $\phi: Q \rightarrow \operatorname{Out}(N)$ is injective and $|\operatorname{Out}(N): \phi(Q)|<\infty$.

If $S=\{1\}$, we get the following corollary whose applications are discussed in Section 3.

Corollary 1.2. For anynon-elementary hyperbolic group $H$ and any finitely presented group $Q$, there exists a short exact sequence (1), where $G$ is hyperbolic and $N$ is a quotient group of $H$. Moreover, $G$ is torsion-free whenever $H$ is.

Remark 1.3. One can generalize Theorem 1.1 to the case where $H$ is a non-elementary group hyperbolic relative to a collection of proper subgroups and $Q$ is finitely generated and finitely presented relative to a collection of subgroups $S_{1}, \ldots, S_{m}$. In this case the collection of peripheral subgroups of $G$ will consist of isomorphic copies of $S_{1}, \ldots, S_{m}$ and the images of the peripheral subgroups of $H$ under the homomorphism $H \rightarrow N$. We leave the proof as an exercise for the reader.

It is also worth noting that an alternative proof of Corollary 1.2 can be obtained by means of Ol'shanskiı̈'s small cancellation theory over ordinary hyperbolic groups [25] without using any results of [28] and [1].

\section{Proof of Theorem 1.1}

Recall that a group $G$ generated by a finite set $X$ is said to be finitely presented with respect to a subgroup $S$ if the kernel of the natural map $\varepsilon: F(X) * S \rightarrow G$, where $F(X)$ is the free group with basis $X$, is the normal closure of a finite subset $\mathcal{R}$ of $F(X) * S$. For instance, any group is finitely presented relative to itself.

Given an element $w \in F(X) * S$, we denote by $|w|_{X \cup S}$ its length with respect to the generating set $X \cup S$. A group $G$ is hyperbolic relative to $S$ if $G$ is finitely presented with respect to $S$ and, in the above notation, there is a constant $C>0$ such that for any $w \in F(X) * S$ such that $\varepsilon(w)=1$, we have

$$
w=\prod_{i=1}^{k} f_{i}^{-1} R_{i}^{ \pm 1} f_{i},
$$


where $R_{i} \in \mathcal{R}$ and $f_{i} \in F(X) * S$ for $i=1, \ldots, k$, and $k \leq C|w|_{X \cup S}$. In particular, this definition becomes the definition of an ordinary hyperbolic group in the case $S=1$.

Let $G$ be a group hyperbolic relative to a subgroup $S$. Given a subgroup $H \leq G$, we denote by $H^{0}$ the set of all elements $g \in H$ of infinite order such that $g$ is not conjugate to an element of $S$. For every $g \in G^{0}$, there exists a unique maximal virtually cyclic subgroup $E_{G}(g)$ of $G$ containing $g$ [29]. Moreover,

$$
E_{G}(g)=\left\{f \in G: f^{-1} g^{n} f=g^{ \pm n} \text { for some } n \in \mathbb{N}\right\} .
$$

In this paper we use the notion of a suitable subgroup of a relatively hyperbolic group introduced in [28]. The most convenient form of the definition is proposed in [1]: A subgroup $H \leq G$ is called suitable if $H^{0} \neq \emptyset$ and

$$
\bigcap_{g \in H^{0}} E_{G}(g)=\{1\} \text {. }
$$

In particular, (3) and (4) imply that every suitable subgroup $H \leq G$ has trivial centralizer.

The key ingredient of the proof of Theorem 1.1 is the following result obtained in [28] (see Theorem 2.4 and its proof there).

Theorem 2.1. Let $G_{0}$ be a group hyperbolic relative to $S \leq G_{0}, H$ a suitable subgroup of $G_{0}$. Then for every finite subset $T=\left\{t_{1}, \ldots, t_{k}\right\} \subset G_{0}$, there exist elements $w_{1}, \ldots, w_{k} \in H$ such that the quotient group

$$
G=G_{0} /\left\langle t_{1} w_{1}, \ldots, t_{k} w_{k}\right\rangle,
$$

satisfies the following conditions:

(i) the restriction of the natural homomorphism $\xi: G_{0} \rightarrow G$ to $S$ is injective;

(ii) $G$ is hyperbolic relative to $\xi(S)$;

(iii) if $G_{0}$ is torsion free, then $G$ is torsion free;

(iv) $\xi(H)$ is suitable in $G$;

Proof of Theorem 1.1. Suppose that $X \subset Q$ is a finite subset generating $Q$. Since $Q$ is finitely generated and finitely presented with respect to $S$, the group $S$ is also finitely generated by [30, Theorem 1.1]. Let $Z$ denote a finite generating set of $S$. Assume also that the kernel of the natural homomorphism $F(X) * S \rightarrow Q$ is the normal closure of a finite subset $\mathcal{R} \subset F(X) * S$. The free product $G_{0}=F(X) * S * H$ is hyperbolic relative to $S$ [30, Theorem 2.40].

It is well known that every non-elementary hyperbolic group $H$ contains a unique maximal normal finite subgroup $K \leq H$, in fact $K$ is precisely the kernel of the $H$ action on the boundary of $H$. Thus passing to the quotient $H / K$ if necessary we may 
assume that $H$ has no nontrivial finite normal subgroups. Since $H$ is non-elementary, it is a suitable subgroup of $G_{0}$ and we may apply Theorem 2.1 to the set

$$
T=\left\{x y x^{-1}, x^{-1} y x \mid x \in X \cup Z, y \in Y\right\} \cup \mathcal{R},
$$

where $Y$ denotes a finite generating set of $H$. Let $G$ be the corresponding quotient of $G_{0}$. By $N$ we denote the image of $H$ in $G$. Clearly (5) and (6) imply that $N$ is normal in $G$ and $G / N \cong Q$. Assertions (b)-(d) of the theorem follow immediately from Theorem 2.1.

To prove (e) we first choose a non-elementary hyperbolic group $L$ with property (T). Ol'shanski1's results [25] (see also [26]) imply that there is a non-elementary hyperbolic common quotient $H_{1}$ of $H$ and $L$. Thus replacing $H$ with $H_{1}$, we may assume that $H$ has property (T), and hence so does $N$. By part (iv) of Theorem 2.1, $N$ is a suitable subgroup, therefore (3) together with the definition of a suitable subgroup implies that $N$ has trivial centralizer in $G$. In particular, there is a canonical injection $Q \rightarrow \operatorname{Out}(N)$. Now (e) follows as in [6, Section 5], and for completeness we sketch a proof below.

Recall the definition of a relatively hyperbolic group given in [8, Definition 1]: if $\mathscr{E}$ is a conjugacy-invariant family of infinite finitely generated subgroups of a group $\Gamma$, then $\Gamma$ is called hyperbolic relative to $\mathcal{E}$ if $\Gamma$ acts isometrically and properly discontinuously on a complete locally compact hyperbolic metric space $\mathcal{X}$ such that each point of the ideal boundary of $\mathcal{X}$ is either a conical limit point or a bounded parabolic point of $\Gamma$, and the elements of $\mathcal{E}$ are precisely the maximal parabolic subgroups of $\Gamma$.

To continue the proof note that if $S$ is infinite, then $G$ is hyperbolic relative to $S$ in the sense of the above definition. Indeed, as we mentioned before, since $G$ is finitely generated, so is $S$ [30, Theorem 1.1], and in this situation the above definition of Bowditch is equivalent to the definition introduced in [30], which is the definition we follow in this paper. The equivalence of the definitions is explained in appendix to [30]. If $S$ is finite, then $G$ is hyperbolic [30, Corollary 2.41], so $G$ acts isometrically, properly discontinuously and cocompactly on a locally compact complete hyperbolic space $\mathcal{X}$.

Precomposing the inclusion $N \hookrightarrow G$ with automorphisms of $N$, we get a sequence of injective homomorphisms from $N$ to $G$, which defines a sequence of $N$-actions on $\mathcal{X}$. These homomorphisms fall into finitely many $G$-conjugacy classes, else a standard rescaling argument of Bestvina-Paulin [7], [31], applied to the $N$-actions on $\mathcal{X}$, produces a nontrivial action on an $\mathbb{R}$-tree which contradicts the fact that $N$ has property (T). Since $N$ has trivial centralizer in $G$, the canonical homomorphism $G \rightarrow \operatorname{Aut}(N)$ in injective, and by the previous sentence its image has finite index in $\operatorname{Aut}(N)$. Let $G_{*}$ be the intersection of all the conjugates of (the image of) $G$ in $\operatorname{Aut}(N)$. Note that $G_{*}$ is a finite index normal subgroup of $\operatorname{Aut}(N)$ that contains $N$. The kernel of the surjection of $\operatorname{Out}(N)=\operatorname{Aut}(N) / N$ onto the finite group $\operatorname{Aut}(N) / G_{*}$ is $G_{*} / N$. Since $G_{*} / N \leq G / N \leq \operatorname{Out}(N)$, we conclude that the image of $Q=G / N$ has finite index in $\operatorname{Out}(N)$. 


\section{Applications}

The idea behind many applications of the Rips construction is to lift a (usually pathological) property of $Q$ to $G$, and the extra control over $N$ provided by Theorem 1.1 makes the job easier.

Prescribing linear representation. Here we build a hyperbolic group that has the same set of $n$-dimensional complex representations as a given finitely generated group; thus representation theory fails to detect hyperbolicity.

Theorem 3.1. For any finitely generated group $\Gamma$ and any integer $n>0$ there is a non-elementary hyperbolic group $G$ and an epimorphism $G \rightarrow \Gamma$ such that every representation $G \rightarrow \mathrm{GL}_{n}(\mathbb{C})$ factors as the composition of the epimorphism $G \rightarrow \Gamma$ and a representation $\Gamma \rightarrow \mathrm{GL}_{n}(\mathbb{C})$.

Proof. First, we build a finitely presented group $Q$ and an epimorphism $Q \rightarrow \Gamma$ such that any representation $Q \rightarrow \mathrm{GL}_{n}(\mathbb{C})$ factors through $Q \rightarrow \Gamma$. Let $\langle S \mid R\rangle$ be a presentation of $\Gamma$ where $S=\left\{s_{1}, \ldots, s_{m}\right\}$ and $R=\left\{R_{1}, \ldots, R_{k}, \ldots\right\}$. The representation variety $\operatorname{Hom}\left(\Gamma, \mathrm{GL}_{n}(\mathbb{C})\right)$ is the algebraic subvariety of the product of $m$ copies of $\mathrm{GL}_{n}(\mathbb{C})$ defined by the relators $R_{1}, \ldots, R_{k}, \ldots$ More precisely, $\operatorname{Hom}\left(\Gamma, \mathrm{GL}_{n}(\mathbb{C})\right)$ is the set of $m$-tuples of elements of $\mathrm{GL}_{n}(\mathbb{C})$ that are mapped to the identity matrix by each $R_{k}$. Let $V_{k}$ be the algebraic variety defined in the product of $m$ copies of $\mathrm{GL}_{n}(\mathbb{C})$ by the first $k$ relators $R_{1}, . ., R_{k}$, and let $I_{k}$ be the corresponding ideal (produced via Hilbert's Nullstellensatz). Since polynomial rings over $\mathbb{C}$ are Noetherian, the chain of ideals $I_{1}, \ldots, I_{k}, \ldots$ stabilizes, i.e. $I=I_{k}$ for some $k$, where $I$ is the ideal that corresponds to $\operatorname{Hom}\left(\Gamma, \mathrm{GL}_{n}(\mathbb{C})\right.$ ) (again via Hilbert's Nullstellensatz). Hence the group $Q:=\left\langle s_{1}, \ldots, s_{m} \mid R_{1} \ldots R_{k}\right\rangle$ has the same representation variety as $\Gamma$, i.e. the inclusion $\operatorname{Hom}\left(\Gamma, \mathrm{GL}_{n}(\mathbb{C})\right) \hookrightarrow \operatorname{Hom}\left(Q, \mathrm{GL}_{n}(\mathbb{C})\right)$ induced by the epimorphism $Q \rightarrow \Gamma$ is a bijection. In other words, any representation of $Q$ into $\mathrm{GL}_{n}(\mathbb{C})$ factors through $Q \rightarrow \Gamma$.

Next goal is to build a non-elementary hyperbolic group with no nontrivial homomorphisms into $\mathrm{GL}_{n}(\mathbb{C})$. It has been known for some time (see [18]) that there exists a non-elementary hyperbolic group $K$ such that any homomorphism $K \rightarrow \mathrm{GL}_{n}(\mathbb{C})$ has finite image. In fact, by [18] any proper quotient of a cocompact lattice in $\operatorname{Sp}(r, 1)$ with $r \geq 2$ has this property, and by small cancellation theory there are proper quotients that are non-elementary hyperbolic. (Kapovich actually proves that $K \rightarrow \mathrm{GL}_{n}(\mathbb{F})$ has finite image for any field $\mathbb{F}$ ). On the other hand, Lubotzky observed in [21, Proposition 1.3] if a group $L$ has the property FAb (which means that every finite index subgroup of $L$ has finite abelianization), then for each $n$ there is a finite index subgroup $L_{n}$ of $L$ such that for any representation $\rho: L_{n} \rightarrow \mathrm{GL}_{n}(\mathbb{C})$ the image $\rho\left(L_{n}\right)$ has connected closure. Lattices in $\operatorname{Sp}(r, 1)$ have FAb, because their finite index subgroups have property (T). Taking $L$ to be any cocompact lattice in $\operatorname{Sp}(r, 1)$ with 
$r \geq 2$, we get $L_{n}$ such that $\rho\left(L_{n}\right)$ has connected closure for any $\rho: L_{n} \rightarrow \mathrm{GL}_{n}(\mathbb{C})$. Let $K_{n}$ be a non-elementary hyperbolic proper quotient of $L_{n}$, so that $\rho\left(K_{n}\right)$ is finite and has connected closure, and therefore, is trivial.

Finally, if $G$ is the hyperbolic group produced by the Rips construction as in Corollary 1.2 with input $H=K_{n}$ and $Q$ as in the first paragraph of the proof, then any representation of $G$ into $\mathrm{GL}_{n}(\mathbb{C})$ factors through the epimorphism $G \rightarrow Q \rightarrow \Gamma$.

Remark 3.2. We do not know whether in Theorem 3.1 one can choose the same $G$ for all $n$. The following representation-theoretic properties of a group involve its complex representations of all dimensions. A finitely generated group $\Gamma$ is called

- representation rigid if for every $n$ there are only finitely many pairwise nonisomorphic $n$-dimensional irreducible representation $\Gamma \rightarrow \mathrm{GL}_{n}(\mathbb{C})$,

- representation superrigid if there is a uniform upper bound on the dimension of the Zariski closure of $\rho(\Gamma)$ for all complex representations $\rho$ of $\Gamma$.

For brevity we just say rigid and superrigid. According to [3] any superrigid group is rigid. The Margulis superrigidity theorem implies that irreducible lattices in semisimple groups of higher rank are superrigid, and the same holds for lattices in $\operatorname{Sp}(n, 1)$ and $F_{4}^{(-20)}$ [11], [15]. Examples of non-linear rigid groups that are not superrigid can be found in [3]. However, we do not know of any examples of finitely presented rigid groups that are not superrigid. (In the earlier version of this paper [5], we incorrectly assumed that such examples were constructed in [3], and we are grateful to Laszlo Pyber and David Fisher who independently found the mistake). As is explained in [5], if $Q$ is a finitely presented rigid group that is not superrigid, and if $K$ is a non-elementary hyperbolic proper quotient of a cocompact lattice in $\operatorname{Sp}(r, 1)$ with $r \geq 2$, then the Rips construction as in Corollary 1.2 with input $H=K$ and $Q$ produces a non-elementary hyperbolic group $G$ that is rigid but not superrigid.

Prescribing minimal actions on Hadamard spaces. A $G$-action on a complete CAT(0) space $X$ is called minimal if $X$ is the only non-empty convex $G$-invariant subspace. Let $\mathscr{H}$ be a class of complete CAT(0) spaces for which there exists a nonelementary hyperbolic group $H$ with the property any isometric $H$-action on a space $X$ in $\mathscr{H}$ has a non-empty fixed-point-set $X^{H}$. Let $G$ be a hyperbolic group produced via the Rips construction as in Corollary 1.2 with input $H, Q$.

Corollary 3.3. Any (isometric) minimal $G$-action on every $X$ in $\mathscr{H}$ factors through the surjection $G \rightarrow Q$. Thus $G$ and $Q$ have the same set of minimal actions on spaces in $\mathscr{H}$.

Proof. Since $N$ is a quotient of $H$, its fixed-point-set $X^{N}$ is non-empty, and also convex by [10, Corollary II.2.8]. The subspace $X^{N}$ is stabilized by $G$ because $N$ 
is normal in $G$, hence $X^{N}=X$ since the $G$-action is minimal. Thus $N$ lies in the kernel of the $G$-action.

Remark 3.4. The simplest example to which Corollary 3.3 applies is when $H$ is any Kazhdan hyperbolic group, and $\mathscr{H}$ is the class of $\mathbb{R}$-trees. Note that the $G$-action is stable if and only if the $Q$-action is stable. In particular, Corollary 3.3 implies that studying stable actions of hyperbolic groups on $\mathbb{R}$-trees is no easier than studying stable actions of finitely presented groups on $\mathbb{R}$-trees. Of course, Corollary 3.3 also has a relatively hyperbolic version, that shows in particular that stable actions of finitely generated relatively hyperbolic groups on $\mathbb{R}$-trees can be as pathological as stable actions of finitely generated groups on $\mathbb{R}$-trees.

Here is a list of some other pairs $(\mathscr{H}, H)$ to which Corollary 3.3 applies.

- $H$ is any Kazhdan hyperbolic group, and $\mathscr{H}$ is the class of affine Hilbert spaces, and cube (or more generally zonotopal) CAT(0) cell complexes [23], [16];

- $\mathcal{H}$ is the class of Hadamard manifolds (i.e. complete simply-connected Riemannian manifolds of nonpositive sectional curvature), and $H$ is the torsion-free Kazhdan hyperbolic group constructed in [14], [17] such that any $H$-action on a manifold in $\mathscr{H}$ has a fixed point;

- finite products of the spaces listed above provided the $H$-action preserves the product structure (which is automatically true in most cases), and fixes a point in every factor.

Adding higher powered relations to hyperbolic groups. A well-known "hyperbolic philosophy" suggests that adding higher powered relations to a hyperbolic group does not change the group too much. For instance if $G$ is non-elementary hyperbolic and $g$ is an element of infinite order, than $G /\left\langle\left\langle g^{n}\right\rangle\right\rangle$ is also non-elementary hyperbolic for all but finitely many $n[25$, Theorem 3$]$.

Recall that a group is called large if it contains a finite index subgroups admitting an epimorphism onto a non-abelian free groups. In a recent paper [20], Lackenby showed that for any large group $G$ and any element $g \in G$, the quotient $G /\left\langle\left\langle g^{n}\right\rangle\right\rangle$ is large for infinitely many $n$. Moreover, in case $G$ is free and non-abelian, the words "infinitely many" can be replaced with "all but finitely many". (A short group theoretic proof of these facts can be found in [27].) One may guess that the same is true if $G$ is a large hyperbolic group and $g$ is an element of infinite order. (It is an easy exercise to construct counterexamples in case we do not require $G$ to be hyperbolic or allow the order of $g$ to be finite.) However, we have the following.

Corollary 3.5. There exists a large torsion-free hyperbolic group $G$ and an element $g \in G$ such that $G /\left\langle\left\langle g^{n}\right\rangle\right\rangle$ is not large for all odd $n \in \mathbb{N}$. 
Proof. Let us consider a short exact sequence (1), where

$$
Q=\left\langle a, x, y \mid a^{-1} x a=y, a^{-1} y a=x\right\rangle
$$

and $N$ has property (T). Observe that the subgroup $S$ generated by $a^{2}, x, y$ has index 2 in $Q$ and is isomorphic to $\mathbb{Z} \times F_{2}$, where $F_{2}$ is the free group of rank 2 . Thus $Q$ is large and hence so is $G$.

Let $K=G /\left\langle\left\langle g^{n}\right\rangle\right\rangle$, where $g$ is a preimage of $a$ in $G$ and $n$ is odd. Suppose that a finite index subgroup $L$ of $K$ admits an epimorphism $\varepsilon: L \rightarrow F$, where $F$ is a non-abelian free group. Clearly $L$ splits into the short exact sequence $1 \rightarrow U \rightarrow$ $L \rightarrow V \rightarrow 1$, where $U$ is a finite index subgroup of a quotient of $N$ and $V$ is a subgroup of $Q /\left\langle\left\langle a^{n}\right\rangle\right\rangle \cong \mathbb{Z} / n \mathbb{Z} \times \mathbb{Z}$. Since property (T) is inherited by quotients and subgroups of finite index, $U$ has property (T). In particular $\varepsilon(U)=1$ and hence the image of $L$ in $F$ is isomorphic to a quotient group of the abelian group $\left.Q /\left\langle a^{n}\right\rangle\right\rangle$, which is a contradiction.

In [13, Lemma 12.2], Dunfield and Thurston showed, among other things, that the property to have virtually positive first Betti number is inherited by all but finitely many quotients of type $G /\left\langle\left\langle g^{n}\right\rangle\right\rangle, n \in \mathbb{N}$, where $G$ is a certain amalgam of finite groups and $g$ is an element of infinite order. Motivated by some long-standing conjectures in 3-dimensional topology, they asked [13, Question 11.1] if this is true for arbitrary amalgam of finite groups and any element of infinite order. Recall that every amalgam of finite groups is hyperbolic. The next corollary shows that the Dunfield-Thurston result can not be generalized to the case of arbitrary hyperbolic groups.

Corollary 3.6. There exists a torsion-free hyperbolic group $G$ and an element $g \in G$ such that $\beta_{1}(G)>0$, but $G /\left\langle\left\langle g^{n}\right\rangle\right\rangle$ has property $(\mathrm{T})$ for all $n \in \mathbb{N}$. In particular, $G /\left\langle\left\langle g^{n}\right\rangle\right\rangle$ contains no subgroups of finite index with positive first Betti number.

Proof. It suffices to consider a short exact sequence (1), where $Q$ is infinite cyclic and $N$ has property (T). If $g$ is a preimage of a nontrivial element of $Q$ in $G$, then for any $n \neq 0, G /\left\langle\left\langle g^{n}\right\rangle\right\rangle$ is an extension of a quotient of $N$ by a finite group. Hence $G /\left\langle\left\langle g^{n}\right\rangle\right\rangle$ has property (T) whenever $n \neq 0$. Hence $G$ has no finite index subgroups with infinite abelianization.

Recursively recognizable properties. A group-theoretic property $P$ is called $r e$ cursively recognizable if there is an effective algorithm that decides from a finite presentation of a group whether or not the group has the property $P$ (or more formally, $P$ is called recursively recognizable if the set of finite presentations of groups with property $P$ is recursive).

Recall that the isomorphism problem is decidable for torsion-free hyperbolic groups [35], [12]. On the other hand many problems are known to be undecidable for hyperbolic groups [4], [22]. For instance, the property to be generated by 
a given number of elements is recursively unrecognizable in the class of hyperbolic groups [4]. Here we provide some other examples. Let $\mathcal{P}$ be a property of groups. We consider the following conditions.

(1) $\mathcal{P}$ is not recursively recognizable in the class of all finitely presented groups.

(2) $\mathcal{P}$ is preserved under taking quotients.

(3) If groups $A$ and $B$ have $\mathcal{P}$, then any extension of $A$ by $B$ has $\mathcal{P}$.

(4) There exists a non-elementary hyperbolic group with property $\mathcal{P}$.

Example 3.7. Kazhdan's property (T) and Serre's property FA satisfy (2)-(4). To show they are not recursively recognizable in the class of all finitely presented groups it suffices to note that (T) implies FA [36] and the free product $\mathbb{Z} / 2 \mathbb{Z} * G$ has property (T) (or FA) if and only if $G \cong\{1\}$. Since the property "to be trivial" is not recursively recognizable in the class of all finitely presented groups, the properties (T) and FA are not recursively recognizable in the class of all finitely presented groups.

Corollary 3.8. If $\mathcal{P}$ is a property of groups satisfying (1)-(4), then $\mathcal{P}$ is not recursively recognizable in the class of all torsion-free hyperbolic groups.

Proof. Let $H$ be a non-elementary hyperbolic group with property $\mathcal{P}$. For every finitely presented group $Q$, we construct a short exact sequence $1 \rightarrow N \rightarrow G_{Q} \rightarrow$ $Q \rightarrow 1$ as in the theorem. By (2) and (3), the group $G_{Q}$ has $\mathcal{P}$ if and only if $Q$ has $\mathcal{P}$. Since $G_{Q}$ is hyperbolic and $\mathcal{P}$ is not recursively recognizable in the class of all finitely presented groups, the result follows.

Kazhdan groups and their automorphisms. By a result of Paulin [32], the group $\operatorname{Out}(H)$ is finite for every hyperbolic group $H$ with Kazhdan property (T). Answering a question of Paulin, Ollivier-Wise [24] constructed Kazhdan groups whose outer automorphism groups are infinite. In fact, they showed that any countable group $Q$ embeds into $\operatorname{Out}(N)$ for some Kazhdan group $N$. In particular, $\operatorname{Out}(N)$ can fail to be finitely presentable (in case $Q$ is not recursively presented). As was noted in [6], this result of Ollivier-Wise implies that any finitely presented group $Q$ can be embedded as a finite index subgroup into $\operatorname{Out}(N)$ for some Kazhdan group $N$.

Our methods allow us to recover (and generalize) these results.

Corollary 3.9. For any finitely generated group $Q$ there is split extension $N \rtimes Q$ that is hyperbolic relative to $Q$, and such that the canonical map $\phi: Q \rightarrow \operatorname{Out}(N)$ is injective, $|\operatorname{Out}(N): \phi(Q)|<\infty$, and $N$ has Kazhdan property $(\mathrm{T})$. In particular, any countable group embeds into $\operatorname{Out}(N)$ for some group $N$ with Kazhdan property $(\mathrm{T})$.

Proof. It suffices to apply Theorem 1.1 for a finitely generated group $Q$, subgroup $S=Q$, and a non-elementary hyperbolic group $H$ with property (T). The assertion "in particular" follows from the fact that any countable group embeds into a finitely generated one. 
Question 3.10. If $H$ is a hyperbolic centerless group with property (T), then $\operatorname{Aut}(H)$ is a finite extension of $H$ [32], so $\operatorname{Aut}(H)$ is also a hyperbolic group with property (T). Hence the automorphism tower of $H$ consists of hyperbolic groups with property (T). Does the tower terminate in finitely many steps? This is true if $H$ is a centerless finite group by a classical result of Wielandt [34, 13.5.4].

\section{Acknowledgements}

We are grateful to William Goldman for discussions on representation varieties, and to the referee for editorial remarks.

\section{References}

[1] G. Arzhantseva, A. Minasyan, D. Osin, The SQ-universality and residual properties of relatively hyperbolic groups. J. Algebra 315 (2007), 165-177. Zbl 05201680

[2] H. Bass, A. Lubotzky, Nonarithmetic superrigid groups: counterexamples to Platonov's conjecture. Ann. of Math. (2) 151 (2000), 1151-1173. Zbl 0963.22005 MR 1779566

[3] H. Bass, A. Lubotzky, A. R. Magid, S. Mozes, The proalgebraic completion of rigid groups. Geom. Dedicata 95 (2002), 19-58. Zbl 1059.20036 MR 1950883

[4] G. Baumslag, C. F. Miller, III, and H. Short, Unsolvable problems about small cancellation and word hyperbolic groups. Bull. London Math. Soc. 26 (1994), 97-101. Zbl 0810.20025 MR 1246477

[5] I. Belegradek, D. Osin, Rips construction and Kazhdan property (T). Preprint 2007. arXiv:math.GR/0605553

[6] I. Belegradek, A. Szczepański, Endomorphisms of relatively hyperbolic groups. With an appendix by Oleg V. Belegradek. Internat. J. Algebra Comput., to appear; preprint 2007. arXiv:math.GR/0501321

[7] M. Bestvina, Degenerations of the hyperbolic space. Duke Math. J. 56 (1988), 143-161. Zbl 0652.57009 MR 0932860

[8] B. Bowditch, Relatively hyperbolic groups. Preprint 1999. www.warwick.ac.uk/ masgak/preprints.html

[9] M. R. Bridson, F. J. Grunewald, Grothendieck's problems concerning profinite completions and representations of groups. Ann. of Math. (2) 160 (2004), 359-373. Zbl 1083.20023 MR 2119723

[10] M. R. Bridson, A. Haefliger, Metric spaces of non-positive curvature. Grundlehren Math. Wiss. 319, Springer-Verlag, Berlin 1999. Zbl 0988.53001 MR 1744486

[11] K. Corlette, Archimedean superrigidity and hyperbolic geometry. Ann. of Math. (2) 135 (1992), 165-182. Zbl 0768.53025 MR 1147961 
[12] F. Dahmani, D. Groves, The isomorphism problem for toral relatively hyperbolic groups. Preprint 2006. arXiv:math.GR/0512605

[13] N. M. Dunfield, W. P. Thurston, The virtual Haken conjecture: Experiments and examples. Geom. Topol. 7 (2003), 399-441. Zbl 1037.57015 MR 1988291

[14] M. Gromov, Random walk in random groups. Geom. Funct. Anal. 13 (2003), 73-146. Zbl 01971826 MR 1978492

[15] M. Gromov, R. Schoen, Harmonic maps into singular spaces and $p$-adic superrigidity for lattices in groups of rank one. Inst. Hautes Études Sci. Publ. Math. 76 (1992), 165-246. Zbl 0896.58024 MR 1215595

[16] F. Haglund, F. Paulin, Simplicité de groupes d'automorphismes d'espaces à courbure négative. In The Epstein Birthday Schrift, Geom. Topol. Monogr. 1, Geom. Topol. Publ., Coventry 1998, 181-248 (electronic). Zbl 0916.51019 MR 1668359

[17] H. Izeki, S. Nayatani, Combinatorial harmonic maps and discrete-group actions on Hadamard spaces. Geom. Dedicata 114 (2005), 147-188. Zbl 1108.58014 MR 2174098

[18] M. Kapovich, Representations of polygons of finite groups. Geom. Topol. 9 (2005), 1915-1951. Zbl 05002573 MR 2175160

[19] I. Kapovich, D. T. Wise, The equivalence of some residual properties of word-hyperbolic groups. J. Algebra 223 (2000), 562-583. Zbl 0951.20029 MR 1735163

[20] M. Lackenby, Adding high powered relations to large groups. Preprint 2007. arXiv:math.GR/0512356

[21] A. Lubotzky, Some more non-arithmetic rigid groups. In Geometry, spectral theory, groups, and dynamics, Contemp. Math. 387, Amer. Math. Soc., Providence, RI, 2005, 237-244. Zbl 1084.22007 MR 2180210

[22] C. F. Miller, III, Decision problems for groups-survey and reflections. In Algorithms and classification in combinatorial group theory (Berkeley, CA, 1989), Math. Sci. Res. Inst. Publ. 23, Springer-Verlag, New York 1992, 1-59. Zbl 0752.20014 MR 1230627

[23] G. Niblo, L. Reeves, Groups acting on CAT(0) cube complexes. Geom. Topol. 1 (1997), 1-7. Zbl 0887.20016 MR 1432323

[24] Y. Ollivier, D. T. Wise, Kazhdan groups with infinite outer automorphism group. Trans. Amer. Math. Soc. 359 (2007), 1959-1976. Zbl 05120622 MR 2276608

[25] A. Yu. Olshanskii, On residualing homomorphisms and $G$-subgroups of hyperbolic groups. Internat. J. Algebra Comput. 3 (1993), 365-409. Zbl 0830.20053 MR 1250244

[26] - On the Bass-Lubotzky question about quotients of hyperbolic groups. J. Algebra 226 (2000), 807-817. Zbl 0957.20026 MR 1752761

[27] A. Yu. Olshanskii, D. V. Osin, Large groups and their periodic quotients. Proc. Amer. Math. Soc., to appear; preprint 2006. arXiv:math.GR/0601589

[28] D. V. Osin, Small cancellations over relatively hyperbolic groups and embedding theorems. Preprint 2004. arXiv:math.GR/0411039

[29] D. Osin, Elementary subgroups of relatively hyperbolic groups and bounded generation. Internat. J. Algebra Comput. 16 (2006), 99-118. Zbl 1100.20033 MR 2217644 
[30] D. V. Osin, Relatively hyperbolic groups: intrinsic geometry, algebraic properties, and algorithmic problems. Mem. Amer. Math. Soc. 179 (843) (2006). Zbl 1093.20025 MR 2182268

[31] F. Paulin, Topologie de Gromov équivariante, structures hyperboliques et arbres réels. Invent. Math. 94 (1988), 53-80. Zbl 0673.57034 MR 0958589

[32] F. Paulin, Outer automorphisms of hyperbolic groups and small actions on R-trees. In Arboreal group theory (Berkeley, CA, 1988), Math. Sci. Res. Inst. Publ. 19, SpringerVerlag, New York 1991, 331-343. Zbl 0804.57002 MR 1105339

[33] E. Rips, Subgroups of small cancellation groups. Bull. London Math. Soc. 14 (1982), 45-47. Zbl 0481.20020 MR 0642423

[34] D. J. S. Robinson, A course in the theory of groups. Second ed., Grad. Texts in Math. 80, Springer-Verlag, New York 1996. Zbl 0836.20001 MR 1357169

[35] Z. Sela, The isomorphism problem for hyperbolic groups. I. Ann. of Math. (2) 141 (1995), 217-283. Zbl 0868.57005 MR 1324134

[36] Y. Watatani, Property T of Kazhdan implies property FA of Serre. Math. Japon. 27 (1982), 97-103. Zbl 0489.20022 MR 0649023

[37] D. T. Wise, A residually finite version of Rips's construction. Bull. London Math. Soc. 35 (2003), 23-29. Zbl 1027.20014 MR 1934427

Received May 18, 2006

I. Belegradek, School of Mathematics, Georgia Institute of Technology, Atlanta,

GA 30332-0160, U.S.A.

E-mail: ib@math.gatech.edu

D. Osin, Department of Mathematics, The City College of the City University of New York, 138th Street and Convent Ave., New York, NY 10031, U.S.A.

E-mail: denis.osin@gmail.com 